\title{
Civilisations
}

Revue internationale d'anthropologie et de sciences

humaines

56 | 2007

Après la catastrophe

\section{L'argent, la famille, les amies : ethnographie contemporaine des tontines africaines en contexte migratoire}

Jeanne Semin

\section{(2) OpenEdition}

\section{Journals}

Édition électronique

URL : https://journals.openedition.org/civilisations/636

DOI : $10.4000 /$ civilisations. 636

ISSN : 2032-0442

Éditeur

Institut de sociologie de l'Université Libre de Bruxelles

\section{Édition imprimée}

Date de publication : 1 décembre 2007

Pagination : 183-199

ISBN : 2-87263-016-3

ISSN : 0009-8140

\section{Référence électronique}

Jeanne Semin, «L'argent, la famille, les amies : ethnographie contemporaine des tontines africaines en contexte migratoire », Civilisations [En ligne], 56 | 2007, mis en ligne le 01 décembre 2010, consulté le 21 septembre 2021. URL : http://journals.openedition.org/civilisations/636 ; DOI : https://doi.org/ 10.4000 /civilisations.636

Ce document a été généré automatiquement le 21 septembre 2021.

(c) Tous droits réservés 


\title{
L'argent, la famille, les amies : ethnographie contemporaine des tontines africaines en contexte migratoire
}

\author{
Jeanne Semin
}

1 En 2006, les remises d'argent des migrants vers leurs pays d'origine auraient atteint à l'échelle mondiale 220 milliards de dollars. Ces transferts se situent aujourd'hui au deuxième rang des transactions financières internationales après celles liées au commerce du pétrole. Les flux migratoires qui les alimentent deviennent donc un enjeu crucial. Sur le total, l'Afrique subsaharienne reçoit une part très limitée, environ 8,1 milliards de dollars dont une petite partie vient de l'Europe, singulièrement de la France (Tasca et al.: 2007). Par ailleurs, le montant des remises financières et sociales des migrants africains dépassant l'aide publique au développement, la contribution des immigrés aux finances de leur pays d'origine fait l'objet de tentatives de récupération politique, notamment à travers l'usage du terme "co-développement $»^{1}$. Enfin, la forte implication des jeunes noirs dans les émeutes des banlieues, révélée par des études statistiques et sociologiques (Lagrange et Oberti 2006), a aussi attiré l'attention sur les problématiques liées à l'intégration de cette «deuxième génération » dans la société française.

2 Cet ensemble de considérations invite les chercheurs en sciences sociales, et en particulier les africanistes, à s'interroger sur les systèmes de relations économiques et symboliques qui donnent 'sens et puissance' à l'immigration africaine aujourd'hui. Des chercheurs tels que Marcel Mauss, Marshall Sahlins ou encore Pierre Bourdieu, ont démontré que tout échange économique comporte un coefficient de sociabilité, et que l'appréhender en termes exclusivement matériels reviendrait à méconnaître la raison d'être de l'échange. Dans notre civilisation caractérisée communément par le règne de l'argent, les remises des migrants africains sont régies par ces valeurs sociales et affectives de l'échange. 
3 De quoi s'agit-il ? Viviana Zelizer définit ces transactions intimes comme autant de " circuits qui incorporent chacun une certaine structure institutionnelle, des manières de s'entendre et des pratiques communes, des relations interpersonnelles qui les distinguent des autres circuits » $(2001$ : 143). Catherine Quiminal (1991) et Christophe Daum (1998) ou encore Patrick Gonin (2006) ont bien montré, concernant la vallée du fleuve Sénégal, principal foyer d'émigration africaine vers la France, que les circuits financiers issus de l'immigration étaient étroitement imbriqués dans une structure de la dette collective. L'envoi d'argent à un enfant, une sœur ou une mère par les migrants peut intervenir à la fois comme une justification et comme un dédommagement de leur fuite vers le monde occidental. La parenté, l'alliance et le groupe ethnique sont autant de relations psychoaffectives qui déterminent les transferts. Je choisis pour ma part d'aborder cette question du côté des femmes, un aspect souvent négligé dans les études précédemment citées. Parmi les immigrés installés en France, les femmes sont pourtant devenues majoritaires dans la tranche d'âge comprise entre vingt et cinquante-deux ans (Borel 2006). Actrices sociales à part entière, les femmes originaires de la Vallée du fleuve Sénégal jouent un rôle économique important, notamment dans tout ce qui concerne les stratégies d'alliance et les cérémonies familiales.

Le statut des femmes africaines migrantes est abordé ici par la porte d'entrée d'une forme d'organisation économique qui leur est propre, la tontine, qui consiste à verser collectivement et périodiquement de l'argent à une caisse. L'appellation "tontine » dérive du nom du Napolitain Lorenzo Tonti, qui inventa, au 17e siècle, un système de rente viagère. Elle fut appliquée aux pratiques d'épargne rotative observées en Afrique et ailleurs, notamment en Asie. Le principe de ces pratiques est simple: la somme des cotisations cumulées est remise, à tour de rôle, à chaque participante. Une épargne ou un crédit est ainsi débloqué à chaque tour de tontine au profit d'une bénéficiaire différente. Les tontines féminines sont très développées en Afrique de l'Ouest, où elles correspondent à une séparation marquée entre les sphères d'activité féminine et masculine et sont imbriquées dans les échanges occasionnés par les cérémonies familiales. On peut se demander dans quelle mesure ces pratiques conservent une dynamique et un sens en France, où les structures familiales pourraient se trouver modifiées par le contexte socioéconomique et par la distance avec la famille élargie. Autrement dit, quels sont les enjeux symboliques, économiques, collectifs et individuels de l'organisation de tontines, toujours féminines, en contexte migratoire ? Précisons la démarche méthodologique des enquêtes sur la pratique des tontines en Afrique de l'Ouest et en France, avant de présenter les données fournies par les deux terrains et ce qu'elles nous apprennent sur ces structures de l'échange, les rapports de genre et les voies d'adaptation propres à la migration africaine en France.

\section{Groupes et réseaux tontiniers : comment observer ?}

Pour appréhender les enjeux financiers contemporains imbriqués dans des réalités humaines, sociales et familiales complexes, les méthodes de l'anthropologie doivent aujourd'hui s'adapter à l'observation et à l'analyse d'un fonctionnement en réseaux. La méthode de l'ethnographie multi-située impulsée par Marcus (1998) s'impose d'ellemême, face aux connections imaginaires et matérielles des scapes ${ }^{2}$ identitaires et déterritorialisés. Actuellement en phase expérimentale, elle a été utilisée ici, et doit être comprise comme un héritage de la méthode comparative qui consiste à observer un 
même objet dans des contextes apparemment différents, mais aussi comme une invitation sur le terrain à suivre des pistes en différents lieux.

Mon approche anthropologique des tontines de femmes africaines s'est, en tous les cas, constituée sur plusieurs terrains. J'ai abordé pour la première fois les tontines au Sénégal au cours d'une enquête de quatre mois dans un quartier populaire de Saint-Louis en 2001. Je suivais alors les réunions de huit groupes, homogènes par l'âge et la catégorie socioprofessionnelle, comptant chacun entre douze et cent-sept femmes, qui avaient entre vingt-cinq et cinquante ans ${ }^{3}$. J'ai aussi réalisé en 2005 une dizaine d'entretiens et participé à deux tours à Bamako. Mais je développerai ici surtout les résultats d'une enquête de terrain menée avec des migrantes subsahariennes: des Maliennes, des Sénégalaises et une Ivoirienne, vivant en banlieue parisienne.

7 Ce travail, réalisé en 2004, devait au départ avoir pour cadre Montreuil, une agglomération de l'Est parisien. A la Maison des Associations de Montreuil, je repérai neuf organisations de solidarité issues de l'immigration (OSIM), gérées par des femmes africaines. Mais il devint rapidement clair que les tontines ne relevaient pas, en France, de la sphère associative. L'accueil réservé par les associations montreuilloises de femmes africaines a été significatif : « Ce n'est pas ici, Madame, tontine et association, ça n'a rien à voir, ce n'est pas ici»; ou au mieux, "ah ... tiens..., je n'avais jamais pensé que l'association pouvait être un lieu de tontine ». Malgré tout, c'est bien en poussant la porte d'une association qui constitue un simple "lieu d'écoute " pour les femmes qu'il a été possible de rencontrer nos informatrices originaires d'Afrique de l'Ouest. Les tontines qu'elles organisent n'ont pas pour cadre l'association, ni même le quartier, mais des réseaux informels de famille, de travail et de classes d'âge. Elles ne sont pas, comme à Saint-Louis, circonscrites à un espace. Le cadre de départ de la ville de Montreuil, défini a priori, était trop étroit pour contenir les réseaux tontiniers observés, qui s'étendent à toute la banlieue parisienne. Cette " réalité éclatée", pour citer Ulf Hannerz (1980), autorise à penser l'approche anthropologique des tontines de la banlieue parisienne en terme de "réseaux ». Les pratiques tontinières observées en France correspondent à ce mode de relations sociales au gré desquelles : « l'espace est de moins en moins un terroir et de plus en plus un lieu de mobilité " (Badie, 1993: 10). Face à ce constat, la méthodologie consista à suivre la piste des actrices dans toute la banlieue parisienne, au fil des réseaux d'échange. Il s'agissait, sur ce terrain discontinu, de partir du vécu et du point de vue des personnes prises dans ces réseaux. Ceux-ci ne peuvent être abordés qu'à partir des « ancrages » en un point particulier de la structure des relations sociales. Outre la participation à deux tours de tontines, les données produites au cours de la deuxième enquête proviennent d'entretiens semi-directifs, individuels ou collectifs, qui ont été menés auprès de dix personnes, dont huit femmes d'origine sénégalaise, malienne ou ivoirienne, et deux hommes sénégalais et malien, qui ont donné leur point de vue sur ces pratiques féminines. Nos observations et les conversations engagées de manière informelle sont aussi particulièrement utiles pour comprendre les enjeux symboliques des pratiques tontinières. Incluons donc, dans la notion de «lieu anthropologique » ou «d'espace social», la possibilité des "parcours qui s'y effectuent, des discours qui s'y tiennent et du langage qui les caractérise » (Augé 1992 : 104).

L'expérience de terrain nous renseigne d'emblée sur une variation formelle des pratiques tontinières entre l'Afrique et la France, laquelle correspond à une adaptation de la structure d'origine à un nouveau contexte où le lien social n'est, semble-t-il, plus 
constitué à partir d'une proximité de voisinage mais à partir d'une communauté d'origine.

\section{Multiples facettes des tontines africaines}

Afin de comprendre les enjeux des tontines dans le cadre de la migration et de pouvoir envisager une analyse comparative, il convient de présenter l'histoire et le fonctionnement de cette pratique tontinière en Afrique de l'Ouest.

10 L'origine des tontines en Afrique fait l'objet de plusieurs interprétations scientifiques qui se conjuguent dans la réalité historique, mais qu'il est intéressant de distinguer ici. De nombreux auteurs (Henry 1991, Lelart 1991, Servet 1995) s'accordent pour penser que la pratique courante des tontines actuelles découle d'une monétarisation des habitudes traditionnelles de collectivisation, et de l'existence, dans les sociétés africaines traditionnelles, de regroupements entre voisins ou entre personnes d'un même groupe d'âge, pour s'entraider à tour de rôle lors des différents travaux (labours, récoltes, restauration des toitures, etc.). Avec les tontines, l'objet «travail» mis traditionnellement en commun serait devenu « monnaie ». Cette hypothèse met l'accent sur une forme d'agir économique, qui privilégie d'une manière générale la collectivisation des ressources pour le bénéfice de chacun. Et ce système de cotisation apparaît comme efficient au Sénégal aussi bien chez les enfants des rues que chez les agriculteurs ou les femmes commerçantes. Ces exigences holistes constituent certainement un terreau fertile pour la cotisation rotative, mais n'expliquent pas l'émergence de groupes structurés et de ces affaires de femmes que sont devenues les tontines ouest-africaines. Abdoulaye Kane (2001: 10) étudie une hypothèse intéressante selon laquelle le développement des tontines en argent correspond au Sénégal à une monétarisation progressive des rapports de dons/contre-dons rituels obligés au sein de la parenté et du voisinage. Ces dons se font en particulier au cours des cérémonies familiales de mariage et de baptême. Ils incombent aux femmes, alors qu'à ces mêmes occasions, les prérogatives religieuses reviennent aux hommes. Au sujet de ces échanges, parfois très dispendieux du fait d'une réciprocité décuplée, les femmes africaines observent une comptabilité précise. La pratique des tontines est étroitement liée à ces dons et contredons occasionnés par les cérémonies de passage: les tontines sont le seul moyen de rassembler les sommes considérables d'argent nécessaires à la satisfaction de ces obligations cérémonielles. La division sexuelle du rituel expliquerait pourquoi les tontines sont, comme le souligne l'adage populaire principalement une affaire de femmes. L'argent est mis en circulation dans un circuit complexe inter-féminin « polarisé par les dépenses cérémonielles » (Moya 2004: 172), où s'enchevêtrent l'activité économique, les dépenses quotidiennes, les formes d'épargne et les dons ou contre-dons.

11 Selon une logique identique à celle mise en évidence par Mauss dans l'Essai sur le don, à propos des échanges rituels haïda et kwakiutl, les femmes africaines risqueraient de "perdre la face», si elles ne satisfaisaient pas à l'obligation de "donner, rendre et recevoir ». La logique contraignante à l'œuvre dans les dons et contre-dons rituels, qui met en jeu l'honneur des femmes impliquées, se retrouve dans les tontines. A Saint-Louis, lors d'un tour de tontine qui réunissait plus de cent femmes, des petites commerçantes âgées d'une quarantaine d'années, je pus d'ailleurs observer que la valeur des cotisations n'était pas fixe. Les participantes versaient dans la calebasse l'exact contre-don de ce qu'elles avaient reçu de la bénéficiaire du jour lorsque c'était leur tour. Si elles n’avaient 
pas encore perçu le tour de la tontine, elles déterminaient elles-mêmes la valeur de leur contribution en fonction de leurs moyens et de leur relation avec la bénéficiaire, chez qui la réunion se déroulait. Cette position, d'après certaines, s'avérait d'ailleurs plus confortable, car elles pouvaient alors choisir la hauteur du défi et ne risquaient pas de se trouver dans une situation où elles ne pourraient pas rendre. Les sommes cotisées, qui variaient entre 250 et 2500 francs CFA, ainsi que les dons en nature collectés dans une bassine à part, étaient soigneusement consignés dans un cahier, et la liste recopiée par la bénéficiaire du tour, qui avait à cœur de pouvoir rendre l'équivalent à ses consœurs lors des tours suivants.

9 décembre 2006 : Tontine de quartier à Niamakoro, Bamako, Mali. La secrétaire inscrit le montant des cotisations de chacune des participantes.

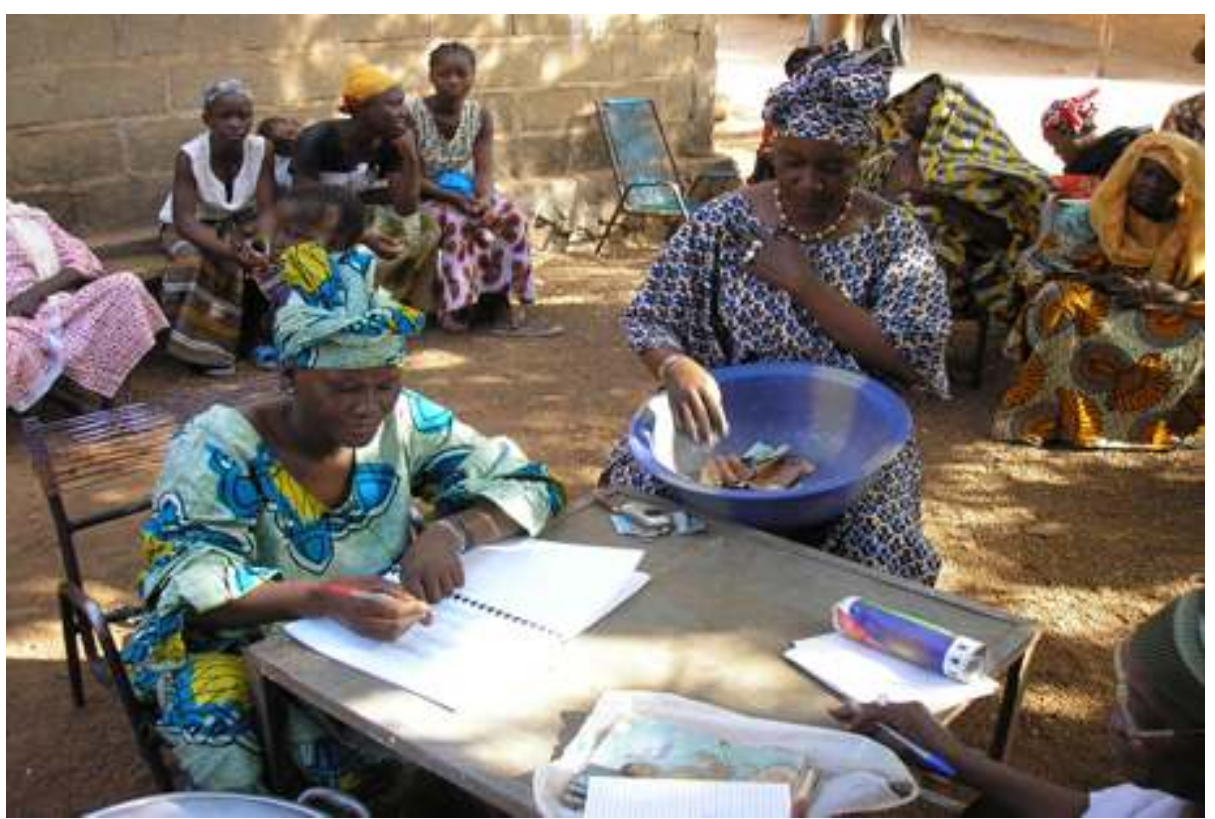

(c) Jeanne Semin

Avec Pierre Bourdieu, on peut également comprendre la force de cette contrainte, pourtant informelle, comme une «dialectique du défi et de la riposte »; la cotisation régulière des membres intervient comme « un défi qui honore celui à qui il s'adresse, tout en mettant à l'épreuve son honneur » (Bourdieu $2000: 31$ ), ou un pari sur l'honneur des autres membres. Cette force contraignante détermine la confiance ${ }^{4}$ et permet l'efficacité économique des tontines. Dans la société saint-louisienne, les groupements de femmes tels que "Deggo bock jom », dont le nom signifie: "Comprendre ensemble la vertu de l'honneur ", sont fondés sur des classes d'âge. Les femmes qui les constituent ont grandi ensemble dans le quartier et partagé les grandes étapes rituelles de leur vie. Les réunions hebdomadaires du groupe constituent un moment d' " entre soi » féminin, où l'on discute des problèmes que l'on partage en tant que femmes (relations matrimoniales, polygynie, santé, éducation des enfants, etc.). Il s'agit d'un échange fondé sur une stricte réciprocité entre personnes de statut égal. Au sein du groupe, on observe un système de relation couplée, nommé Ndey Dické, "Mère Bracelet»: les femmes liées par cette relation déterminée par le hasard d'un tirage au sort se doivent une solidarité renforcée, notamment pour les dons occasionnés par les cérémonies familiales ${ }^{5}$. 
13 L'efficacité économique d'une réciprocité équilibrée au sein de groupes solidaires permet aux femmes africaines de faire face aux exigences des dépenses cérémonielles, mais aussi à la crise économique du développement à partir des années 1980. L'échec général des politiques d'ajustement structurel s'accompagna d'une faillite du système bancaire importé d'Occident au profit de grands chefs religieux ou politiques, qui ne remboursèrent pas les prêts accordés. Les banques apparurent comme peu fiables et inaptes à satisfaire le besoin de mobilisation de l'épargne. En ouolof, depuis lors, l'expression «dama bank» signifie : «je suis ruiné ». Les tontines, en tant que forme plus efficace d'épargne solidaire, connurent alors un essor particulier. D'après les données fournies par des études comparatives réalisées entre 1987 et 1994 dans cinq pays de la zone Franc, (Côte d'Ivoire, Sénégal, Cameroun, Congo et Gabon), le taux moyen de participation à une tontine est passé de $29 \%$ à $70 \%$ de la population (Benoît et al. 1998). La crise qui touche aussi la sphère masculine de l'emploi impose de plus en plus de responsabilité financière aux femmes. Les tontines permettent alors de répondre à un besoin croissant de mobilisation de capitaux. Grâce à une très grande souplesse d'organisation, on observe une grande diversité d'arrangements tontiniers. Michel Lelart l'explique par le fait qu'il n'y a pas de modèle préétabli : « ce ne sont pas les personnes qui s'adaptent à un schéma, c'est la tontine qui s'adapte aux besoins de ses membres » (1991 : 251). Ce système contraignant de cotisation collective apparaît comme généralement plus fiable, plus efficace et plus accessible que le système bancaire anonyme, coûteux, et peu sûr en Afrique.

14 Les économistes, qui s'intéressèrent de très près au phénomène, désignèrent les tontines comme étant des associations rotatives d'épargne et de crédit (AREC). La tontine optimise en effet les disponibilités financières de ses membres, elle opère sur l'épargne un effet de " contrainte sociale à l'auto-contrainte ", d'après l'expression de Norbert Elias (1990: 181). L'obligation contractée au sein de la tontine l'emporte sur l'obligation de solidarité. La tontine permet donc de mettre de côté une épargne qui ne pourrait pas se réaliser autrement dans ce contexte de redistribution incessante des biens. Les organisations non gouvernementales et les institutions de développement, ayant remarqué cette capacité des femmes à épargner au sein de leur classe d'âge, les encouragèrent vivement à se constituer en associations avec une présidente, souvent la "Mère » de la tontine, qui avait pris l'initiative de sa création, une secrétaire et une comptable, et un dépôt de statut à la préfecture. L'objectif était de pouvoir contrôler ces groupements et de les encourager à investir les sommes accumulées dans des activités génératrices de revenus. Les femmes réunies en groupements, reconnus comme unités efficaces de gestion, se virent accorder des micro-crédits beaucoup plus facilement que les hommes. Elles remboursaient collectivement en usant de cette même force contraignante à l'œuvre dans les cérémonies et dans les tontines. Ainsi les femmes africaines furent-elles beaucoup plus soutenues que les hommes dans leurs initiatives économiques.

15 Malgré les recommandations des ONG, la tontine est fréquemment mobilisée pour satisfaire à des obligations rituelles, lesquelles sont présentées dans le discours idéal comme un gaspillage ostentatoire. Pour contourner le problème, les groupements constituent souvent à côté de l'épargne rotative une caisse de secours spécifiquement réservée au paiement de ces échanges cérémoniels, baptisés " problèmes sociaux ». Dans une très belle conférence prononcée en 1997, Marshall Sahlins développait ainsi l'idée selon laquelle les sociétés locales du Tiers-monde, loin de laisser anéantir leurs systèmes de valeurs culturels sous l'effet d'une globalisation homogénéisante, organisent les forces 
dominantes $\mathrm{du}$ système capitaliste en fonction de leurs propres systèmes de représentation, utilisent les richesses du capital pour l'expansion des fêtes et de la politique, afin de subvenir à la parenté (Sahlins 1997 : 15). Pour la pratique des tontines, on peut identifier deux stratégies: d'une part, renforcer les investissements d'ordre social et symbolique (dans la politique d'alliance, les obligations de solidarité, etc.), et d'autre part, permettre des investissements d'ordre économique et matériel (accumulation d'un capital en vue de sa fructification, acquisition de mobilier électroménager, amélioration des conditions de vie). Les deux types de stratégie ont des résultats profondément imbriqués. Les jeux d'alliance et de solidarité communautaire peuvent être intéressés, et servir la satisfaction de besoins matériels ou l'amélioration des conditions de vie, et inversement, le jeu économique d'accumulation peut n'être qu'un moyen pour renforcer les alliances et valeurs traditionnelles de l'honneur. A travers les tontines, le capital social et symbolique se convertit en capital économique; l'ensemble constitue une forme précieuse de richesse et une garantie face aux aléas de la vie.

16 La souplesse des tontines et leur capacité à conjuguer la satisfaction des contraintes coutumières et modernes s'observent à travers une diversification importante de ces pratiques féminines. Le vocable "tontine», en français, recouvre une diversité de pratiques parfois désignées sous des termes différents dans les langues maternelles de leurs organisatrices. En bambara, par exemple, le ton désignera généralement le principe associatif, et donc naturellement un tour de tontine impliquant réunion des membres et relation directe entre les cotisants.

9 décembre 2006 : Tontine à Bamako, Mali. Les femmes du quartier de Niamakoro se réunissent chaque samedi depuis 15 ans

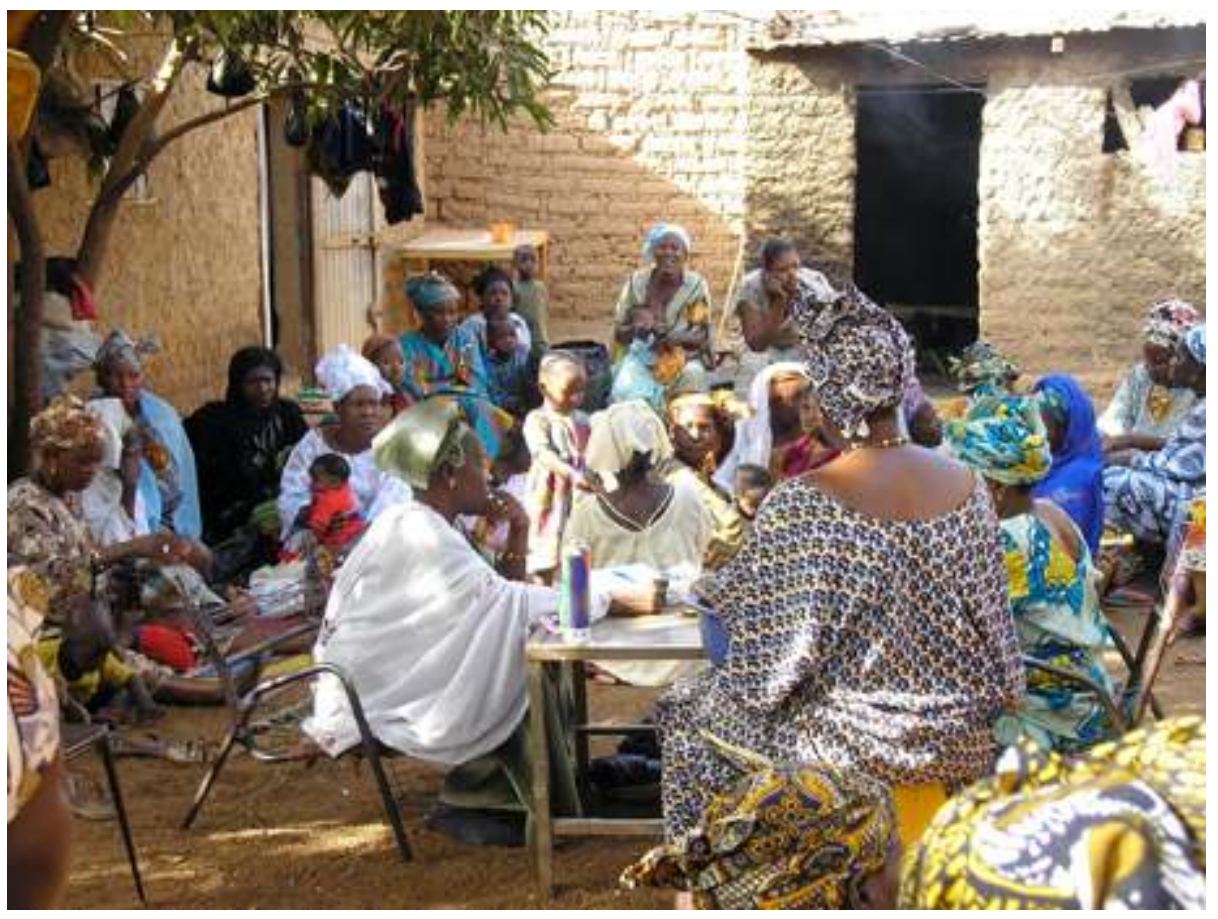

(c) Jeanne Semin

17 Le pari ${ }^{6}$ se rapporte plus à la cotisation auprès d'une gérante qui collecte les parts et les redistribue, sans que cela implique une rencontre directe entre les cotisant(e)s, malgré 
tout lié(e)s par la dette. Il est possible de distinguer trois grands types de cotisations rotatives que l'on retrouve sous d'innombrables variantes:

- les tontines qui reposent sur un principe associatif et donnent lieu à une réunion périodique des membres, qui se retrouvent pour cotiser. On les appelle : « tour de tontine ${ }^{7}$,

- les cotisations périodiques organisées exclusivement pour soutenir les membres en cas de problème ou de cérémonies familiales coûteuses, que l'on appellera ici « caisses de secours » : l'argent n'est plus alors distribué à tour de rôle, mais lorsque l'un des membres doit participer à une cérémonie familiale,

- et enfin les tontines qui ne donnent pas lieu à réunion, pour lesquelles les membres n'ont pas de relations directes entre eux, et qui sont organisées par une gérante : on les appellera " cotisations auprès d'une gérante ".

Certaines tontines peuvent être mixtes et recouper divers types. L'utilisation du terme «tontine " pour désigner l'ensemble de ces pratiques entraîne une confusion, exprimée ainsi par l'une de mes informatrices en banlieue parisienne :

- Tontine, c'est pari, non? Mais il y en a qui parlent aussi de tontine pour les tons,

tu vois...

- Non, moi j'appelle ça caisse.

- Caisse ? Même si l'argent tourne?

- Non, ça, c'est pari...

\section{Tours de tontine en banlieue parisienne}

Le contexte d'immigration en France impulse une nouvelle dynamique d'adaptation des tontines féminines africaines. Les pratiques tontinières perdurent à travers la migration et sont même relativement dynamiques. Toutes les Africaines qui en ont les moyens participent à au moins une tontine, et souvent à plusieurs. Marianne explique par exemple :

La pratique des tontines est donc tout aussi dynamique en France qu'au Sénégal. Dans un contexte qui n'est plus celui d'une crise économique et bancaire, quelles sont alors les raisons qui poussent les femmes africaines à pérenniser leurs pratiques tontinières audelà de la migration? Au travers de la description de deux tours de tontines en banlieue parisienne, on tâchera de percevoir les enjeux des tontines en contexte migratoire, après avoir dégagé différences et points communs avec les tontines observées en Afrique de l'Ouest.

Un samedi, Bintou m'invite au tour de son amie d'enfance, Anta, une Sénégalaise d'environ quarante ans, venue en France pour rejoindre son mari, dont elle a aujourd'hui divorcé. Elle a trois enfants et travaille comme vendeuse dans un magasin africain au nord de Paris. Bintou et Anta ont grandi ensemble à Dakar. Anta est entrée cette année dans le tour de tontine de la famille matrilinéaire élargie d'une collègue de travail. C'est la première fois qu'elle organise le tourchez elle, et elle a fait beaucoup de dépenses. Elle a convié sa voisine malienne; sa filleule, qui était là pour aider aux préparatifs, a aussi invité deux amies. Les huit femmes qui participent de plein droit au tour arrivent vers seize heures, avec leurs invitées. Elles sont habillées de splendides boubous, et très maquillées. Elles habitent toutes à l'autre bout de Paris. A dix-sept heures, Anta sert le déjeuner : " riz à la cubaine », un plat très riche avec des boulettes de viande hachée, des œufs, des gambas, et du poulet, que nous partageons dans un plat commun, assises sur des nattes. Puis nous installons le salon pour qu'il y ait la place de danser, car un concours 
de "petits pagnes" est prévu. Il s'agit d'un moment de danse effrénée où les participantes de la tontine montrent aux autres leurs dessous sénégalais brodés : celle qui porte le plus beau " petit pagne » sera récompensée par un lot de produits de beauté. Une leçon de séduction, dont nous ne développerons pas ici les enjeux. Après avoir bien dansé, les huit femmes donnent leurs cotisations à la présidente : cinquante-cinq euros chacune, dont huit fois cinquante sont immédiatement reversés à Anta, et huit fois cinq sont gardés pour une caisse de secours, au cas où l'une des membres du groupe aurait un "problème social » à régler. Anta sert à nouveau des collations, on danse, les jeunes montrent les nouvelles danses... Anta n'a pas gagné beaucoup, car sur les quatre cents euros qu'elle reçoit, elle en a dépensé deux cents pour les préparatifs, mais ce n'était pas le but: "Le tour d'aujourd'hui c'était surtout pour s'amuser, on a dansé, on a mis les bijoux... jusqu'à dix heures du soir. C'est pour qu'on oublie un peu des choses. On s'amuse!» Khoudia, quant à elle, pense que les tours de tontines sont une occasion de se retrouver entre femmes africaines: "comme on ne se voit pas souvent ici en France!» Ces moments de distraction sont en tous les cas réservés aux femmes, comme le précise Coumba : «I y a des Sénégalaises et des Maliennes, mais que des femmes, ah, là il n'y a jamais d'homme !».

Grâce à l'invitation de Rokia, j'ai pu assister un autre samedi au tour de la «tontine des jeunes ». Selon elle, ce ton a pour objectif de : «montrer les coutumes aux jeunes qui sont nées ici ». Il s'organise au sein d'un réseau familial, les membres du groupe sont toutes soninke et font partie d'une même parentèle élargie, celle des Sylla. Elles habitent très loin les unes des autres. Fatou, mariée et mère de trois enfants, accueille le tour, elle habite dans un grand ensemble d'HLM en zone 3, près d'une station de RER, ce qui constitue une bonne raison de faire les réunions chez elle, bien que ce ne soit pas son tour. Rokia et moi arrivons les premières, Fatou nous apprend d'emblée que deux personnes ont téléphoné pour se décommander. Pour nous faire patienter, la maîtresse de maison glisse dans le magnétoscope une cassette vidéo du mariage d'une de ses amies, et on discute de mariages, de l'activité des griottes ... Une participante téléphone pour dire qu'elle ne viendra pas, elle s'est trompée de jour, elle habite trop loin. Une autre appelle encore: elle ne peut pas venir, la sœur de son mari est chez elle. Diawara, une des participantes, arrive quelques heures plus tard. Elle est habillée dans un grand boubou et porte des bijoux en or. Elle traîne une petite valise à roulettes, remplie de marchandises à vendre. La réunion est l'occasion pour Diawara de montrer les tissus africains et l'encens qu'elle a à vendre. Pour elle, les tontines sont son "petit monde ", c'est là qu'elle fait ses affaires. Elle en a une autre le lendemain et une autre le samedi suivant. Puis vient Lala, une jeune fille de dix-neuf ans, née ici, mariée depuis ses dix-sept ans. Elle est la sœur de Fatou. De confession wahhabite, elle porte le voile islamique. $\mathrm{Ce}$ «ton » réunit normalement quinze personnes, mais ce jour-là seulement quatre membres sont présentes. Les cotisations sont fixes : quinze euros par mois. Mais les participantes ne se voient que tous les deux mois, elles versent donc trente euros à chaque réunion. Trois cents euros reviennent à l'une d'entre elles, et le reste des cotisations est gardé dans une caisse, dont le contenu sera utilisé pour organiser une fête. Cette semaine, les absentes passeront chez Fatou pour régler leur dette. La première qui demandera à recevoir le tour sera la gagnante, les quatre personnes qui sont présentes ont déjà reçu leur part. Lala est la seule des jeunes nées en France et engagées dans cette tontine à être présente. Fatou, Rokia et Diawara sont nées au Mali. Diawara explique : "Cette tontine, c'est pour les jeunes, mais elles sont un peu tête en l'air. Avec la tontine de la famille de mon mari ce n'est pas comme ça, les femmes sont toujours là!». Face à l'absentéisme croissant des jeunes filles, Fatou et Rokia parlent de mettre en place un pari, les jeunes n'auraient pas à se déplacer et Fatou serait la gérante. 
Elles souhaitent conserver le principe d'une caisse permettant d'organiser des fêtes pour entretenir la coutume, les habits de fête et les danses.

\section{Stratégies économiques et identitaires d'adaptation}

La description de ces deux tours nous apprend plusieurs choses. Tout d'abord les tontines restent en France un moment d'«entre soi » féminin bien distinct de la sphère masculine; il est hors de question que les hommes y participent. Ceux-ci organisent de leur côté des " caisses communautaires villageoises ", dont le fonctionnement a été étudié par Christophe Daum (1991) puis Catherine Quiminal (1998). D'après Abdoulaye Kane, qui réalisa une enquête auprès des migrants hal-pulaar originaires de la vallée du fleuve Sénégal, certaines caisses communautaires ayant pour fondements la caste ou l'ethnie ont une section masculine et une section féminine, mais la participation aux tontines reste exclusivement féminine (Kane 2001 : 136). En contexte migratoire, comme au Sénégal, les rapports de genre s'affirment dans des relations de réciprocité et des compétitions ostentatoires, à travers une division sexuelle des rôles.

J'ai pu relever également, dans les points communs entre les tontines organisées en France et en Afrique de l'Ouest, la forte imbrication des tontines dans la ronde des échanges rituels. Lors des réunions, les événements matrimoniaux et les organisations de baptêmes occupent une place importante dans les discussions. C'est aussi une façon pour les femmes de se tenir informées des alliances et des naissances dans la parentèle élargie, et de mobiliser les parentes et amies autour de ces événements pour lesquels il est jugé important d'être très entourées et de pouvoir mobiliser beaucoup d'invités. L'organisation de caisses de secours est aussi fréquente en France. L'argent est épargné et chaque membre peut demander à recevoir le contenu de la caisse pour la célébration d'un mariage ou d'un baptême dans sa famille. Anta, par exemple, verse dix euros par mois : "L'autre tontine, ça, c'est avec la famille. Tous les mois on cotise, on met l'argent dans une caisse. On met la caisse à la banque. Quand il y a un mariage, ou un baptême où tu en as besoin, tu le dis, on te donne mille euros ». Le sujet est aussi polémique qu'au Sénégal dans les discussions entre femmes. Coumba, par exemple, critique ce système: elle associe les frais de cérémonies et les dons et contre-dons rituels à du " gaspillage » et affirme que les caisses ne servent à rien et qu'il est plus important de cotiser pour un projet concret. Si le but de l'ostentation est d'assurer la publicité des actes ou des statuts sociaux, son effet est de détourner une partie de l'argent à des fins improductives, mais n'est-elle pas nécessaire à la pérennisation du système tontinier lui-même?

On retrouve en France le dynamisme des trois grands types de cotisations observés au Sénégal et au Mali. Le ton des jeunes Soninke que nous avons décrit ci-dessus par exemple était en train d'évoluer vers un pari sans réunion, du fait de la faible mobilisation des jeunes pour les rencontres. Bintou, qui m'avait invitée au tour d'Anta, aime beaucoup l'ambiance des tours, mais ne trouve pas non plus le temps d'y participer chaque mois et d'en organiser, elle cotise donc cent cinquante euros auprès d'une gérante en faisant juste passer une enveloppe :

$\mathrm{Au}$ cours des entretiens, j'ai pu constater que ce type de tontine était aussi développé en France qu'au Sénégal. Elles satisfont des besoins qui sont avant tout financiers et les sommes mobilisées sont en général plus importantes. Avec sa tontine, Bintou peut percevoir trois mille euros, qu'elle utilise tous les deux ans pour partir au Sénégal, payer le billet d'avion et les frais sur place. 
Il semble donc que les tontines en France remplissent les mêmes objectifs qu'au Sénégal : mobilisation rapide de capitaux pour des investissements dans les cérémonies familiales, mobilisation de capitaux plus importants pour la réalisation de projets personnels, pérennisation des liens sociaux, des coutumes et de la sphère féminine d'affirmation de soi. Cependant, il faut peut-être insister sur ce dernier point en contexte français. En effet, d'après les observations d'Abdoulaye Kane, l'apparition historique de tontines de femmes africaines en France correspond, au départ, surtout à un besoin de sociabilité. Celles qui arrivèrent en France à partir des années 1970, pour rejoindre leurs époux dont l'immigration était devenue plus durable, supportaient parfois mal l'inexistence quasigénérale de rapports sociaux intenses dans le cadre du quotidien et du voisinage. Elles demeuraient souvent enfermées chaque jour dans leur appartement, en attendant le retour du travail de leur époux. La barrière de la langue et de la culture les condamnait aussi à l'isolement. Abdoulaye Kane rapporte quelques exemples de femmes africaines migrantes basculant dans la folie, du fait, selon lui, de cet isolement peu habituel dans leur milieu d'origine (Kane 2001 : 141). A travers les tontines, les femmes se rencontrent, échangent leurs vues sur les problèmes communs, partagent les informations relatives aux services sociaux avec lesquels elles traitent, s'entraident dans leur émancipation de l'emprise masculine. Les tontines apparaissent ainsi comme des cadres de socialisation collective pour les femmes, qui les utilisent en contexte migratoire pour s'adapter à leur nouvel environnement. Pour les jeunes de la "deuxième génération ", la situation n'est pas la même, le sentiment d'exclusion et les besoins de sociabilité sont différents. Les aînées ont pourtant à cœur de transmettre une tradition féminine africaine à leurs filles.

A Saint-Louis, les tontines correspondaient avant tout à une solidarité de quartier et de classe d'âge. En France, les facteurs de la parenté et de l'ethnie sont très importants, plus, d'ailleurs, que celui de la nationalité; c'est ainsi qu'Abdoulaye Kane observe que les femmes hal-pulaar originaires de la Mauritanie et du Sénégal se retrouvent plus facilement dans une tontine simple qu'avec les Ouolof ou les Soninke, qui sont pourtant de la même nationalité (Kane 2001 : 138). Les tontines se sont organisées avant tout au gré de relations établies au pays et des réseaux de la parenté, de l'ethnie, ou de la communauté d'origine. Les cercles de sociabilité s'entrecroisent et se combinent. Les gérantes, qui organisent des cotisations impersonnelles, recrutent aussi leurs «clientes » dans les trois cercles de la parenté élargie, du travail et du voisinage. En banlieue parisienne, elles s'associent souvent en vue de cumuler leur capital social et de constituer une tontine plus importante. Aujourd'hui, les tontines s'ouvrent petit à petit à des relations établies en France; l'accès à un travail et les relations de voisinage permettent l'émergence de tontines qui peuvent réunir plusieurs origines nationales ou ethniques. $\mathrm{Si}$ les tontines restent encore majoritairement une affaire de femmes africaines, l'importance du phénomène est remarquable par l'intérêt que lui porte la presse. Dans un journal gratuit, quelqu'un témoignait récemment, en exagérant peut-être un peu: «Dans les cités (...) pratiquement toutes les femmes ont recours à la tontine. Lancée par les femmes d'Afrique de l'Ouest, elle touche maintenant toutes les communautés".

Enfin, la différence majeure entre les tontines africaines en France et en Afrique de l'Ouest réside dans le montant des sommes cotisées, et de ce fait dans les possibilités d'investissement économique ou symbolique qu'elles offrent. A l'issue du tour d'Anta, lorsque j'interrogeai les participantes sur les différences entre leurs pratiques tontinières ici et là-bas, elles répondirent en chœur avec un sourire satisfait : « Ah, il y a des différences, ici c'est mieux, c'est plus luxueux». Lors de ce tour,le premier auquel j'assistais en France, je 
fus en effet étonnée du faste de la réunion, qui était l'occasion d'un repas très copieux et d'une compétition récompensée de lots payés par Anta elle-même. Pour ce type de tontine impliquant réunion, on observe d'ailleurs une compétition ostentatoire dans le luxe des réceptions organisées à tour de rôle par chacune des membres lorsqu'elles reçoivent leur tour de tontine. Cette surenchère suscite des réticences chez certaines. Les cotisations auprès d'une gérante oscillent elles, en France entre cinquante et cinq cents euros par mois, ce qui représente plus de quinze fois le montant des cotisations mensuelles les plus élevées à Diamouguène (10000 CFA). Et lorsqu'on évoque la question, Coumba souligne aussi spontanément : "La principale différence c'est qu'eux, ils n'ont pas tellement les moyens. Parce qu'ici, même ceux qui ont les plus petits moyens font des tontines qui sont plus importantes que les tontines organisées au Sénégal». Si elles ont souvent un compte en banque, les femmes africaines installées en France n'ont pas forcément accès au crédit, et, de fait, n'en ont pas besoin. Coumba, qui est une femme d'affaires, est catégorique: "Jamais! Je n'ai jamais fait de crédit bancaire ». La souplesse de l'organisation d'une tontine leur parait plus accessible et plus "normale ", d'après l'expression de Bintou : "C'est que le crédit dans une banque, on a l'impression d'être dans les difficultés, alors que quand on est dans les tontines, on a l'impression de faire ce qui est normal. Le crédit, ça fait peur, on a l'impression de s'endetter». Les capitaux débloqués peuvent représenter en France des sommes non négligeables. Pour prendre la cotisation la plus élevée dont j'ai entendu parler sur le terrain, une femme qui cotise cinq cents euros par mois dans un groupe de vingt-cinq membres reçoit de la tontine douze mille cinq cents euros tous les deux ans environs. Catherine est arrivée en France à l'âge de huit ans, elle vit en concubinage, cumule deux emplois et investit l'argent de cette tontine dans un petit commerce en Côte d'Ivoire, géré par sa tante maternelle et sa cousine. Aujourd'hui, beaucoup des femmes maliennes et sénégalaises rencontrées choisissent d'investir leur épargne indépendamment de leur époux, dans la construction d'un commerce ou d'une maison dans leur pays d'origine.

\section{Rapports économiques au sein de la parenté}

Envisageons dès à présent ces investissements économiques au sens étymologique d' oikonomia :«l'art d'administrer une maison». Dans un contexte africain, la racine oikos (maison) pourrait revêtir le sens de " concession ", qui signifie autant le lieu d'habitation que l'unité familiale qu'elle abrite. Si, du fait des migrations, il n'y a plus de coïncidence entre unité résidentielle et cellule familiale, la concession, qui revêt aujourd'hui des allures de réseau, est toujours le lieu d'une économie, qui devient donc internationale. Le terme nomos se rapporte, lui, à la notion de convenances, ou de normes, observées dans l'administration de cette maison. Ces convenances que l'on a tendance à percevoir comme réglées de manière figée, sont en fait l'objet de la mise en œuvre de stratégies, plus ou moins calculées, par les acteurs sociaux.

31 Dans le cadre des tontines féminines observées en banlieue parisienne, les discussions abordaient souvent la question des rapports économiques au sein du couple et celle de la polygamie. Dans la grande majorité des sociétés d'Afrique de l'Ouest, le lien matrimonial est réglé par une répartition budgétaire des responsabilités conjugales. Dans ce cadre, les convenances (nomos), la coutume et, pour certains, la loi coranique, veulent que le mari prenne en charge les besoins matériels élémentaires de sa (ses) femme(s) et de ses enfants. Chaque mois, il doit donner à sa femme la «ration», et chaque jour la 
" dépense ». Il doit aussi prendre en charge le coût du logement et acheter des vêtements. Sur les sociétés régies par une division prononcée entre les sphères d'activités féminine et masculine, Jane Guyer précise : « La spécialisation des responsabilités budgétaires n'est jamais totale, elle oscille en fonction des capacités de chaque sexe à faire face à sa propre sphère, et à toucher l'autre sphère et à échanger les responsabilités » (1988:171-172). Pourtant, en contexte migratoire en France, un mari malien me donnait un chiffre tout à fait approximatif mais révélateur : $2 \%$ des femmes maliennes en France participaient selon lui aux frais du foyer, bien qu'elles aient souvent accès à des revenus. Parmi mes informatrices, beaucoup de femmes originaires d'Afrique de l'Ouest refusaient en effet de partager les frais de loyer, les factures, les courses, etc. Le principe sous-jacent à celui de la responsabilité économique des hommes étant celui de la propriété, inaliénable, du produit du travail des femmes, "ce qui est gagné est gagné ", et une part de ce qui est gagné est capitalisé à travers les tontines. En usant ainsi des obligations traditionnelles de leur mari et des avantages de l'accès au revenu (par le travail ou par les allocations familiales), elles s'assurent un capital social et économique d'indépendance, qu'elles présentent surtout comme une sécurité face à la polygamie des hommes :

Dans le cadre des tontines, les femmes africaines s'entraînent et s'entraident, en profitant intelligemment des lois françaises, telles que l'interdiction de la polygamie. Tout cela fait des tontines des cadres de socialisation collective et d'émancipation. Parmi la dizaine de femmes fréquentées assidûment en banlieue parisienne, trois d'entre elles avaient divorcé de leur mari polygame. En 1988, Odile Journet soulignait qu'en contexte d'immigration, les possibilités de recours au divorce pour les femmes de maris polygames étaient réduites, en raison de leur manque d'autonomie économique et de leur isolement social. Quinze ans plus tard, et depuis l'interdiction de la polygamie en France survenue en 1993, on observe un recours de plus en plus fréquent au divorce, ou à la menace de divorce, en cas de velléités polygyniques des maris. En 2005, Anta, forte d'un capital social, familial et économique, a quitté son époux qui, ne l'ayant pas épousée civilement, s'apprêtait à prendre une seconde épouse. Un an plus tard, elle se remariait à la Mairie avec un homme qui acceptait ses conditions et ses enfants. Aujourd'hui, la conjuration de la polygamie et la constitution d'un capital d'indépendance peuvent apparaitre, par certains aspects, comme un des moteurs des pratiques tontinières. C'est une des raisons pour lesquelles la participation des femmes africaines à des tontines se fait parfois à l'insu de leurs époux. Marianne cotise dans six tontines, mais son époux n'a connaissance que d'une seule.

Dans les entretiens, nos interlocutrices évoquent systématiquement leur mère. Bintou, par exemple, parle de sa mère, une commerçante installée à Dakar, qui finance ses affaires d'import-export grâce aux tontines. Elle n'a jamais eu recours aux banques, et elle refuse d'en entendre parler. Malgré la mort de son mari en 1968, elle a pu élever ses enfants et les envoyer faire des études en France et aux Etats-Unis grâce aux tontines. Elle constitue un modèle de réussite aux yeux de Bintou. Le don de l'épargne collectée peut intervenir comme une marque de reconnaissance par rapport à la transmission d'une éducation et de gestes féminins, notamment celui de la cotisation. Lorsque l'épargne tontinière n'est pas envoyée directement en liquide à la mère, elle peut servir à la construction d'une maison près de la concession maternelle, ou à aller lui rendre visite. Diawara, Malienne soninke d'un peu moins de quarante ans, venue en France rejoindre son époux, illustre l'utilité des tontines par une petite histoire. Incapable, faute de moyens, de se rendre au chevet de sa mère malade, elle a demandé à la gérante d'une des 
tontines auxquelles elle participe de lui donner le tour en urgence; elle a donc pu se rendre auprès de sa mère. Avec le tour d'une autre tontine, elle a pu se faire fabriquer des bracelets en or « 24 carats ». Diawara voulait montrer à sa mère que leur séparation avait valu le coup, car même si elle souffrait de la séparation, elle n'était pas dans le besoin. La rupture avec la famille maternelle est vécue douloureusement par les femmes lors de leur mariage. Beaucoup de chants traditionnels la mettent en scène comme un malheur, qui semble plus difficile à surmonter si le mariage entraîne la migration. Si l'épargne tontinière ne profite pas directement à la mère, elle peut aussi être investie dans la branche maternelle de la famille. Coumba par exemple, qui a perdu sa mère, a financé grâce aux tontines un petit commerce au profit de sa famille maternelle.

Ce transfert de liquidités au fil de la dette lignagère ascendante peut causer le désarroi des descendants. Sur le terrain, fréquenter les associations, les points d'écoute dans les banlieues, m'a permis de relever les impressions des jeunes face aux pratiques économiques de leurs mères. D'après les jeunes d'origine africaine rencontrés, leurs mères font toutes des tontines: "De toute façon, les daronnes ${ }^{8}$ maliennes, tous les samedis après-midi, elles sont prises avec leurs tontines». Les jeunes semblent éprouver un certain agacement à l'égard des tontines, ils en discutent entre eux :

Ils se sentent alors exclus et délaissés par leurs mères au profit des tontines et autres affaires de femmes. Durant les réunions de tontines, les enfants, surtout les garçons, sont écartés, car les femmes aiment se retrouver entre elles pour ce moment qui leur appartient. Un jeune se plaignait même parce que sa mère l'envoyait toujours « à droite à gauche » pour faire passer des enveloppes de cotisation, qu'il avait par ailleurs très envie de détourner. Alors qu'il me raccompagnait à l'arrêt du bus, le fils d'une de mes informatrices me tint ce discours :

Le jeune fils d'Awa parlait ainsi, alors que sa mère venait de me dire qu'elle plaçait son épargne tontinière sur un compte pour ses enfants. L'ethnologue n'a pas pour objectif de dégager le vrai du faux, mais de rendre compte des situations telles qu'elles sont vécues par les intéressés. En l'occurrence, l'amertume des jeunes illustre un conflit intergénérationnel sous-jacent entre les membres de familles immigrées d'Afrique de l'Ouest. La représentation du temps et de l'espace, et le choix du territoire investi affectivement et économiquement, divisent la famille dans la conception de l'administration des biens du foyer entre ici et là-bas, en faveur des ascendants ou des descendants.

\section{Conclusion}

L'observation multisites et l'analyse comparative nous permettent donc ici de mettre en lumière d'une part le dynamisme des tontines de femmes africaines en contexte migratoire français, et d'autre part l'adaptation stratégique de leurs formes et fonctions. On fait alors face à la difficulté de décrire et d'interpréter à la fois la continuité et le changement des modes d'organisation et des convenances économiques, sociales et familiales à travers la migration.

Formellement, la pratique des tontines s'adapte et se trouve, à Paris, moins intégrée dans la vie locale que dans les réseaux familiaux et ethniques de sociabilité déterritorialisés qui s'étendent dans toute la banlieue. Au fil des années et de l'intégration des femmes africaines, qui s'installent de plus en plus nombreuses en France, on peut observer dans 
certaines cités l'émergence de tontines de quartier parfois multiethniques. Au sein des circuits et groupes tontiniers, les femmes africaines s'affirment grâce à des rapports de réciprocité interféminins, qui restent bien distincts de la sphère masculine. Ces circuits se caractérisent par la polarisation des événements cérémoniels liés à la parenté, dont les femmes conservent la prérogative, et par la primauté des liens consanguins sur les liens conjugaux. Ceci est observable notamment dans l'usage qui est fait des flux monétaires capitalisés et redistribués, jamais mis en commun avec l'époux. A travers les tontines, les femmes africaines installées en France mettent en œuvre des stratégies individuelles et collectives pour tirer parti à la fois des obligations coutumières de leur époux, qui ont la charge économique du foyer, et des avantages qu'offre la migration en terme d'accès à un revenu régulier. Le dynamisme des tontines s'explique par leur fonction de capitalisation de ces ressources économiques proprement féminines. L'objectif est de se donner les moyens d'une autonomie financière et d'une influence dans les prises de décision les concernant, notamment vis-à-vis de la polygamie de leurs époux. Les tontines permettent également aux femmes africaines installées en France d'affirmer avec plus de force la valeur d'un statut personnel au sein du couple et des réseaux familiaux et ethniques, jusqu'en Afrique. Dans cette perspective, les tontines apportent le soutien indispensable de l'amitié qui permet non seulement de se contraindre à l'épargne, mais aussi se soutenir mutuellement face aux contradictions que peut impliquer la migration. Avec Thomas Faist (2005), on préférera, s'agissant des processus d'adaptation sociale postmigratoire, utiliser le terme d'accommodation, qui souligne le caractère stratégique d'une intégration qui s'accommode des apports et des travers du milieu d'accueil pour la reproduction de formes de sociabilité réinventées et de logiques statutaires perpétuées. Il serait alors intéressant de voir dans quelle mesure la pratique des tontines (sous une forme ou sous une autre) a toujours un sens pour les filles d'origine africaine qui sont nées et ont grandi en France.

\section{BIBLIOGRAPHIE}

APPADURAI, Arjun, 2001. Après le colonialisme. Les conséquences culturelles de la globalisation. Paris, Payot.

AUGE, Marc, 1992. Non-lieux. Introduction à une anthropologie de la surmodernité. Paris, Le Seuil.

Benoit, Kevin, S. Ferass et S. Gourna-Douath, 1998. Les tontines en Afrique. Mémoire de maîtrise réalisé sous la direction de Jean-Paul Azam. Toulouse, Université des sciences sociales.

BADIE, Bertrand, 1993. « Flux migratoires et relations transnationales », Études internationales, 24

(1), pp. 7-16.

BAILly, Anatole, 1950. Dictionnaire Grec Français. Paris, Hachette.

BenVEniste, Émile, 1969. Vocabulaire des institutions indo-européennes. Paris, Les Éditions de Minuit.

BOREL, Catherine, 2006. Enquête annuelle de recensement 2004 et 2005. Près de cinq millions d'immigrés à

la mi-2004. Étude de l'Institut National de la Statistique et des Études Économiques nº 1098. 
BOURDIEU, Pierre, 2000. Esquisse d'une théorie de la pratique. Précédé de trois études d'ethnologie Kabyle. Paris, Le Seuil.

CoRnWALl, Andrea (éd.), 2005. Readings in Gender in Africa. Londres, The International African Institute.

Daum, Christophe (dir.), 1998. « Migrants et solidarité Nord Sud », Hommes et Migrations, n¹214.

DAUM, CHRISTOPHE, 1998. Les associations de Maliens en France. PARIS, KARTHALA.

ELIAS, Norbert, 1991. La société des individus. Paris, Editions Fayard.

FAIST, Thomas, 2006. «Trans-State Social Spaces and Accomodation: A Case of Change in Boundaries of the Political», dans le cadre de la conférence «1985-2005. Vingt ans de recherches sur les migrations internationales ». Poitiers, MAISON DES SCIENCES DE L'HOMME. http://uptv.univpoitier.fr

Gonin, Patrick,

2005. «Jeux d'acteurs et enjeux territoriaux : quelles migrations pour quel développement? L'exemple du bassin du fleuve Sénégal (République du Mali) », in M. Charef et P. Gonin (dir.), Émigrés-Immigrés dans le développement local. Agadir, ORMES/Sud Contact.

2006. "Migrations internationales et développement des pays d'origine », dans le cadre de la conférence «1985-2005. Vingt ans de recherches sur les migrations internationales ». Poitiers, Maison des Sciences de l'Homme. http://uptv.univ-poitier.fr

GUYER, Jane, 1988. «Dynamic Approaches to Domestic Budgeting: Cases and Methods from Africa », in Daisy Dwyer et Judith Bruee (éds.), A Home Divided: Women and Income in the Third World , pp. 155-172. Stanford, Stanford University Press.

HANNERZ, Ulf, 1983 [1980]. Explorer la ville. Éléments d'anthropologie urbaine. Paris, Éditions de Minuit.

HENRY Alain, Guy-Honoré TCHENTE et Philippe GuILleRME-DieUMEGARD, 1991. Tontines et banques au Cameroun, les principes de la Société des amis. Paris, Karthala.

ITEANU, André, 1983. La ronde des échanges : De la circulation aux valeurs chez les Orokaiva. Paris, Editions de la Maison des Sciences de l'Homme.

JoURNET, Odile et Sylvie FAINZANG, 1988. La Femme de mon mari. Anthropologie du mariage polygamique en Afrique et en France. Paris, l'Harmattan.

KANE, Abdoulaye, 2001. Les caméléons de la finance populaire au Sénégal et dans la diaspora: dynamique des tontines et des caisses villageoises entre Thilogne, Dakar et la France. Thèse de Doctorat, Université d'Amsterdam.

LAGRANGE, Hugues et Marco OBERTI, 2006. Émeutes urbaines et protestations, une singularité française. Paris, Les Presses Sciences Po.

Lecour Grandmaison, Colette, 1978. La natte et le manguier. Paris, Mercure de France.

LELART, Michel, 1991. « Les informalités financières : le phénomène tontinier »in J.-L. Lespes Les pratiques juridiques, économiques et sociales informelles, pp. 249-267. Paris, PUF.

MARCUS, George E., 1998. « Ethnography in/of the World System: the Emergence of Multi-Sited Ethnography ", in George E. Marcus (éd.) Ethnography through Thick and Thin, pp. 79-104. Princeton, Princeton University Press.

MARIE, Alain, 1997. L'Afrique des individus. Paris, l'Harmattan. 
MAUSS, Marcel, 1966. «Essai sur le don », in Sociologie et Anthropologie. Paris, PUF.

MoYA, Ismaël, 2004. « Entre démesure et tempérance. Argent et don au féminin à Dakar », in

Marcel Drach (dir.), L'argent. Croyance, mesure, et spéculation. Paris, La Découverte.

Quiminal, Catherine, 1991. Gens d'ici, Gens d'ailleurs. Paris, Christian Bourgeois.

SAHLINS, MARSHALL, 2000. Les Lumières en Anthropologie. Nanterre, Société d'Ethnologie.

SERVET, Jean-Michel (dir.), 1995. Épargne et liens sociaux, études comparées d'informalités financières.

Paris, Aupelf-Uref-Association d'Economie Financière.

TAsCA, Catherine, Jacques Pelletier et Bernard BARRAux, 2007. Le co-développement à l'essai.

Rapport d'information du Sénat français $n^{\circ} 417$.

VAUGHAN, Megan, 1983. "Which Family? Problems in the Reconstruction of the History of the

Family as an Economic and Cultural Unit », in Cornwall Andrea (éd.), 2005, Readings in Gender in

Africa. Londres, The International African Institute.

ZELIZER, Viviana, 2001. « Transactions intimes »,Genèses, 42, PP.121-144.

\section{NOTES}

1. L'usage du terme "co-développement", notamment dans le cadre de la campagne présidentielle de 2007 en France, est caractéristique. Inventé par Sami Naïr en 1997, ce concept recouvre aujourd'hui plusieurs mesure législatives et politiques visant le retour des migrants et le développement économique de leur région d'origine, afin de réduire et maitriser les flux migratoires en France. Cette conception utilitariste du développement paraît discutable, notamment parce qu'elle s'appuie sur une récupération politique de la contribution des immigrés au développement de leur pays d'origine, mais aussi parce qu'elle part de l'a priori, faux, selon lequel le développement des pays d'origine aboutirait à court terme à enrayer les migrations (Daum 1998 a).

2. Le concept de scapes est élaboré par Arjun Appadurai dans son ouvrage sur les phénomènes d'hybridation identitaire dans les dynamiques de la mondialisation. Ces différents paysages se définissent selon lui comme des nouveaux espaces déterritorialisés: "multiples-mondes constitués par les imaginaires historiquement situés de personnes et de groupes dispersés sur toute la planète » (Appadurai $2001: 69$ ).

3. Pour plus de précisions, se référer à la typologie présentée à la page 33 de mon mémoire de maîtrise : «Les pratiques rotatives d'épargne et de crédit à Diamouguène. Etude d'une solidarité communautaire féminine à Saint-Louis du Sénégal », Université Louis Lumière, Lyon 2, 2002.

4. La confiance au sein des tontines est aussi assurée par un ensemble de stratégies mises en œuvre par les gérantes pour amoindrir les risques de défaillance, en donnant par exemple le dernier tour de la tontine à celle dont la réputation est la moins fiable.

5. La relation de solidarité renforcée observée entre Ndey Dické pourrait être comparée à celle que souligne Megan Vaughan entre Chinjira au Malawi (Vaughan : 1983).

6. L'utilisation de ce vocable français en bambara pour désigner un certain type de tontine illustre bien la mise en œuvre d'une logique du défi d'honneur que j'évoquais plus haut.

7. Le tour est un modèle de l'organisation féminine au Sénégal. Les Sénégalaises pratiquent le "tour de thé", le "tour de danse »; elles se rendent les unes chez les autres à tour de rôle, principalement au sein de groupes d'âge. Ce vocable fait également référence au cercle de la danse, figure du rassemblement entre égales, des relations à la fois d'intimité, de licence et de compétition ostentatoire. 
8. Les jeunes des banlieues françaises utilisent ce terme d'argot pour désigner les mères.

\section{RÉSUMÉS}

L'article aborde le versant féminin de l'immigration subsaharienne en France. Installées en France depuis les années 1980-1990, les femmes africaines perpétuent leurs pratiques économiques et en particulier celle des tontines, très dynamiques dans les banlieues parisiennes. Pour comprendre le phénomène, l'auteur, en se basant sur des observations de terrain menées au Sénégal et au Mali et sur l'apport des théoriciens du don, présente tout d'abord les aspects fondamentaux des pratiques de tontines à travers leur développement historique en Afrique de l'Ouest. Au terme d'une analyse comparative, elle montre comment, dans un contexte d'immigration, les tontines ont continué à satisfaire des besoins variés de sécurité, sociale et psychologique, pour les femmes immigrées en France. Elles correspondent à la mise en œuvre de stratégies féminines d'adaptation, qui questionnent l'organisation de l'économie au sein de la famille, les rapports de genres et de générations, en contexte migratoire.

This paper considers the female element in sub-Saharan immigration to France. Settled in France since the 1980s and 1990s, African women perpetuate their economic practices, and in particular the "tontines" (money-go-rounds), which are very dynamic in the Paris suburbs. To understand this phenomenon, the author has carried out field studies in Senegal and Mali, while calling upon the contributions of "gift theorists". Following upon a comparative analysis, she shows how the tontines continue to help meet the varied needs for security, social and psychological, among immigrant women in France. They correspond to the implementation of female coping strategies that question the economic organisation of the family, as well as gender and generational relations, in a migratory context.

\section{INDEX}

Mots-clés : économie, Afrique de l'Ouest, migration, genre, tontines

Keywords : economy, West Africa, migration, gender, tontines

\section{AUTEUR}

\section{JEANNE SEMIN}

Jeanne Semin est aujourd'hui doctorante à l'École pratique des hautes études, Paris-Sorbonne, où elle poursuit ses travaux en anthropologie économique. Après une maîtrise sur les tontines dans les quartiers de Saint-Louis du Sénégal et un Diplôme d'études approfondies sur les pratiques tontinières des femmes originaires d'Afrique de l'Ouest en banlieue parisienne, elle fait porter sa recherche doctorale sur les gestes de cotisation dans une perspective féminine, transnationale, et encastrée dans le social de l'économie ouest africaine. Ce projet est soutenu par une allocation doctorale de la Région île-de-France, et dirigé par Odile Journet-Diallo.

[jeannesemin@hotmail.com] 